\title{
A Non-disruptive Technology for Robust 3D Tool Tracking for Ultrasound-Guided Interventions
}

\author{
Jay Mung ${ }^{1}$, Francois Vignon ${ }^{2}$, and Ameet Jain $^{2}$ \\ 1 University of Southern California \\ 2 Philips Research North America
}

\begin{abstract}
In the past decade ultrasound (US) has become the preferred modality for a number of interventional procedures, offering excellent soft tissue visualization. The main limitation however is limited visualization of surgical tools. A new method is proposed for robust 3D tracking and US image enhancement of surgical tools under US guidance. Small US sensors are mounted on existing surgical tools. As the imager emits acoustic energy, the electrical signal from the sensor is analyzed to reconstruct its $3 \mathrm{D}$ coordinates. These coordinates can then be used for 3D surgical navigation, similar to current day tracking systems. A system with realtime 3D tool tracking and image enhancement was implemented on a commercial ultrasound scanner and 3D probe. Extensive water tank experiments with a tracked $0.2 \mathrm{~mm}$ sensor show robust performance in a wide range of imaging conditions and tool position/orientations. The 3D tracking accuracy was $0.36 \pm 0.16 \mathrm{~mm}$ throughout the imaging volume of $55^{\circ} \times 27^{\circ} \times 150 \mathrm{~mm}$. Additionally, the tool was successfully tracked inside a beating heart phantom. This paper proposes an image enhancement and tool tracking technology with sub-mm accuracy for US-guided interventions. The technology is non-disruptive, both in terms of existing clinical workflow and commercial considerations, showing promise for large scale clinical impact.
\end{abstract}

Keywords: Ultrasound, Tool Tracking, Interventions, Navigation.

\section{Introduction}

X-ray and Ultrasound (US) are ubiquitous for guidance of interventional procedures, with the latter being used more in terms of total procedure numbers. Estimated at over 10 million procedures per year, US-guided interventions range from routine needle insertion for regional anesthesia [1] to biopsies [2] and percutaneous ablation of cancer [3] to more advanced procedures such as structural heart repair 4. Its low cost, ease of use, excellent soft tissue visualization and use of non-ionizing radiation make it a popular modality for both diagnostics and interventions. The biggest limitation however is its lack of robust tool visualization. X-ray, despite its lack of soft tissue contrast and harmful radiation, has hence been the primary modality to fill this gap. With the advent of advanced

G. Fichtinger, A. Martel, and T. Peters (Eds.): MICCAI 2011, Part I, LNCS 6891, pp. 153-160, 2011.

(C) Springer-Verlag Berlin Heidelberg 2011 

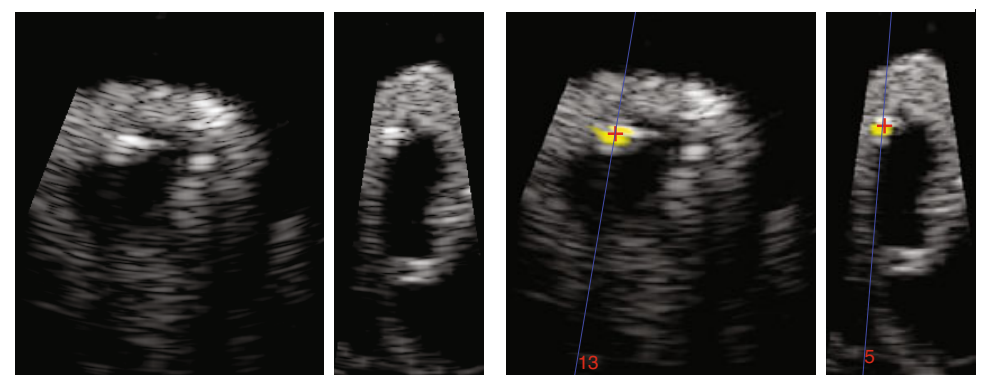

Fig. 1. Left: azimuth and elevation slices through a 3D US image of a catheter in a beating heart phantom. Note the difficulty in tool detection. Right: same, with tool enhancement enabled. Blue lines indicate positions of orthogonal slices. Note that the "unenhanced" images are automatically selected to display the catheter tip.

surgical navigation and image-fusion, research attempts have been made to improve tool visualization. However, these attempts have not yet fully translated to large-scale clinical practice. In particular, external tracking technologies have made some progress at the expense of clinical workflow, leading to slow adoption rates. Clinically viable and robust $3 \mathrm{D}$ tool visualization still remains a challenge.

The "invisible tool" phenomenon is well known in US imaging and interventions (Figure1Left). Several approaches have been proposed to try to overcome this problem. These include roughening the tool surface to make it more isotropically reflective [5]. This has translated clinically [6], but the solution is not yet robust enough for difficult interventions [5]. Ultrasound beamforming protocols have also been modified to better detect straight specular reflectors [7. This also has translated clinically [8]. However such a solution currently works only for a limited category of ultrasound probes (linear or curvilinear geometry), limited needle incidence angles with some a priori knowledge of needle orientation, and inside sub-regions in the tissue image. Doppler imaging of a vibrating tool has also been attempted to enhance tool visualization [9], however requiring changes to the imaging pipeline including a drop in imaging frame rate and interference from moving tissue. Note that all aforementioned technologies only provide image enhancement of the tool but do not yield absolute position information of the tool. External tracking technologies such as optical tracking have been introduced as solutions providing absolute instrument tracking [10. Since these methods alter the existing clinical workflow, they have seen a limited clinical translation, mostly in orthopedic and neurological interventions. A robust solution for US guided interventions is still needed.

A promising direction is to mount a small US "transponder" element on the tool that, upon receiving ultrasound energy from the US beams sent from the imaging probe, re-radiates ultrasound energy toward the probe, thus acting as a very bright reflector. The concept was first introduced in 1984 [1]. At that time, a passive receiver method was also introduced where upon reception, instead of re-radiating ultrasound energy, a low-voltage electrical signal is passed to 
the system for image enhancement. This method was introduced clinically by ATL to enhance needle guidance of breast biopsies [12. Another embodiment was later described by Vilkomerson [13] and used in a similar way for aiding catheter ablation in pigs [14. These systems provided enhanced tool visualization but not position and orientation coordinates of the tracked tools. Furthermore these early accounts made use of 2D US imaging technology which limited the enhancement feature to a plane. For these reasons, they had limited clinical utility. Building upon this technology, an offline technique was later proposed to detect location by analyzing the ultrasound signals received at the tracked transducer from an experimental 3D imaging probe [15]. Though promising, this technique was not integrated with an imaging system for image enhancement and interventional guidance. Alternately, attempts have been made to localize intra-body ultrasound transducers by deploying a number of discrete ultrasound elements to track the tranducer's position via trilateration [1617]. However, these still require a registration of tool position to the US image, making the method difficult to translate clinically.

Key Contributions: As far as the authors are aware, this paper is the first to propose a system for real-time 3D tool tracking and image enhancement on a commercial scanner. It tracks a US sensor by using the US signals impinging on it to compute its $3 \mathrm{D}$ position. The key contributions are:

- A new technique to achieve 3D tool tracking with an accuracy of $0.36 \mathrm{~mm}$

- Intuitive tool image enhancement with automatic 2D slice selection (Fig. 1)

- Extensive validation of a prototype integrated with a commercial scanner

- A solution with potential for clinical implementation

\section{Methods}

Principle of Operation: US sensor(s) are embedded at known locations on the surgical tool. For position tracking, angular position is derived by knowing the angular direction(s) of the US beam(s) that impinge on the receiver, while depth information is derived from the time-of-flight from emission of the respective beam(s). Tracking two receivers can yield orientation of the tool. For image enhancement, (i) an image of the received signals as a function of depth and angle; or (ii) the 3D coordinate can be overlaid on the US image. A schematic description of the principle of operation is provided in Fig. 2 a.

Tracking: A 3D US imaging probe emits ultrasound beams that regularly sample the field of view (FOV) in an array of $N_{A Z} \times N_{E L}$ beams in the azimuth and elevation directions, respectively (the geometry of US acquisition is depicted in Fig. 2b). The temporal signals sensed by the receiver during the acquisition of one image are formatted in a 3D $N_{A Z} \times N_{E L} \times$ time "data matrix". In order to retrieve angular position information with a better resolution than that given by the spacing of the US beams, first a maximum intensity projection (MIP) of the 

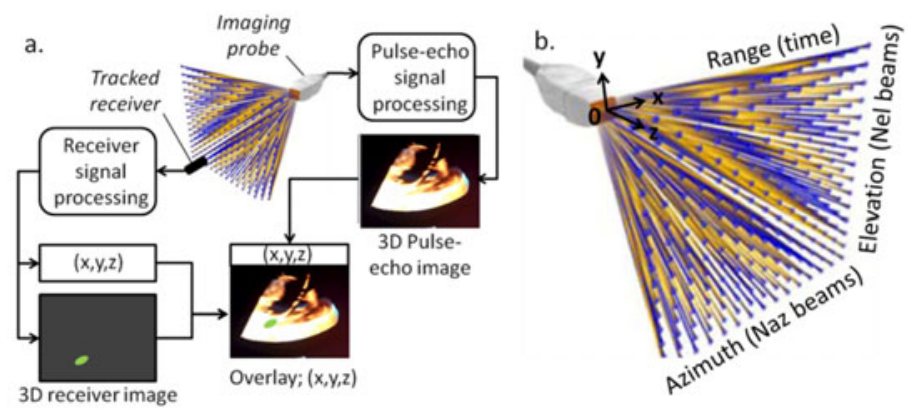

Fig. 2. a: Schematic of principle of operation. b: Depiction of the coordinate systems.

"data matrix" over the time dimension is performed to yield a $2 \mathrm{D}\left(N_{A Z} \times N_{E L}\right)$ MIP matrix on which a 2D Gaussian fit is applied. The Gaussian center is used to estimate the angular coordinates of the receiver in the US coordinate system. The depth information is obtained by finding the time at which the maximum signal arrives at that angle and multiplying by the speed of sound. Thus with angle and range information, the $3 \mathrm{D}$ sensor location is computed.

Display Enhancement: The computed position of the sensor is used to slice through the $3 \mathrm{D}$ pulse-echo data received by the scanner and the 3D image of the receiver, extracting two orthogonal $2 \mathrm{D}$ slices through the sensor - one at constant azimuth and the other at constant elevation angle. These two slices are displayed as an overlay of sensor position (in color) on the standard pulse-echo US image (in grayscale).

Experimental Setup: The 3D imaging X7-2 probe is driven by an iE33 scanner (Philips Healthcare, Bothell, WA) in "Live 3D" mode with a FOV of $\approx 55^{\circ}($ azimuth $) \times 27^{\circ}($ elevation $) \times 150 \mathrm{~mm}$ (range) at an imaging rate of $21 \mathrm{~Hz}$. For this application, the iE33 is equipped with an in-house system that streams the scanner's radiofrequency pulse-echo data to a separate workstation at a lowered frame rate. The US receiver is a PVDF hydrophone with a $0.2 \mathrm{~mm}$ aperture equipped with a 20dB pre-amplifier (model HGL-0200, Onda Corp., Sunnyvale, CA). Sensor acquisitions are synchronized by the iE33 scanner's 'beam out' and 'volume out' trigger signals that mark the start of emission of each US beam and each US volume (an ensemble of $N_{A Z} \times N_{E L}$ beams), respectively. Sensor data is streamed to a workstation at the same rate of the scanner's pulse-echo RF data through a data acquisition card (Gage Applied Technologies, Lachine, Canada). The receiver is suspended in a water bath by a $3 \mathrm{D}$ motorized stage (model MM4006, Newport Corp., Irvine, CA) that is controlled by the central workstation through a GPIB interface. The stage is specified accurate and repeatable to $<5 \mu \mathrm{m}$ and thus provides ground-truth coordinates. A manual rotation component is also available in the $\mathrm{x}-\mathrm{z}$ plane. Streamed data from the scanner and sensor are processed live using Matlab to yield tracked sensor positions and overlay volume $2 \mathrm{D}$ slices with a prototype frame rate of $0.26 \mathrm{~Hz}$. 


\section{$3 \quad$ Experiments and Results}

System Calibration: The first step prior to the validation experiments was to calibrate the system. Data was acquired with the stage translating the receiver in $1 \mathrm{~mm}$ increments over four rectangles in x-y parallel planes at depths of $20 \mathrm{~mm}$, $60 \mathrm{~mm}, 100 \mathrm{~mm}$, and $130 \mathrm{~mm}$. A total of 2000 data points were collected. An unconstrained nonlinear optimization minimizing fiducial registration error calibrated the following variables: speed of sound (SOS) in water, transmit beam angles in water, and time offset between the imaging system 'beam out' trigger and US probe beam emission. The calibrated SOS was $1491.6 \mathrm{~m} / \mathrm{s}$. These parameters were used for the subsequent validation experiments.

\subsection{Tracking Accuracy}

Tracking Accuracy Over the 3D US FOV: The first experiment evaluated the system accuracy throughout the extent of the 3D US FOV. The system acquired data in the same manner as the calibration step (Fig. 31), collecting a total of 5110 data points. $10 \%$ of the points were chosen at random to calculate a registration matrix using Procrustes analysis. The registration matrix was then applied to the remaining $90 \%$ of the points, from which Target Registration Error (TRE) values were calculated. The $10 \%$ random point selection and registration was repeated 100 times to provide a total of 459,900 TRE values. The TRE values were then grouped by slice depth and azimuth angle, with the results shown in Fig. 4. The accuracy is $0.36 \pm 0.16 \mathrm{~mm}$ across the FOV with no significant biases in any one direction. Furthermore, there is no significant correlation to the azimuth angle. The $2.1 \%$ distance error at $20 \mathrm{~mm}$ separation suggests a $1.2^{\circ}$ mean rotation accuracy using two sensors separated by that distance.

Accuracy as a Function of Depth: A second experiment was performed to evaluate the system accuracy throughout depth at the center of the US FOV. The experimental protocol is identical to the first experiment except that the
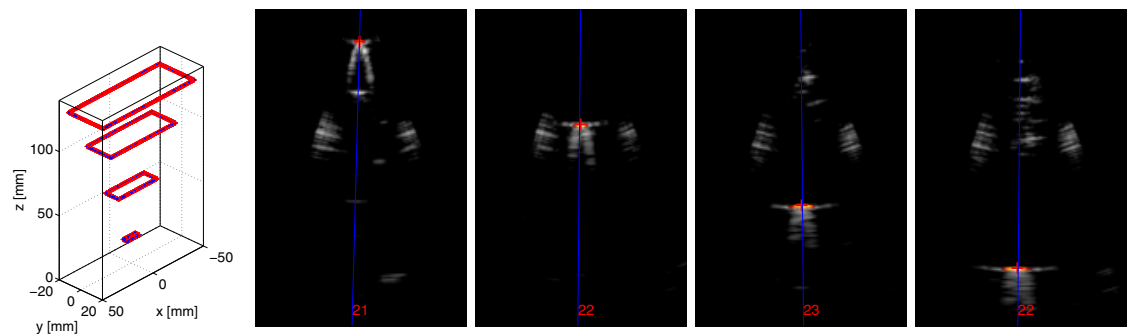

Fig. 3. Left: the tracked sensor ground truth and estimated positions (red crosses and blue stars, respectively). Right: sample overlay images ('hot' colormap receiver images with grayscale pulse-echo images) at the four rectangle depths $(20 \mathrm{~mm}, 60 \mathrm{~mm}, 100 \mathrm{~mm}$, $130 \mathrm{~mm}$ ). Shown are 4 automatically selected azimuth slices through $3 \mathrm{D}$ datasets. The resolution degrades with increasing depth. 

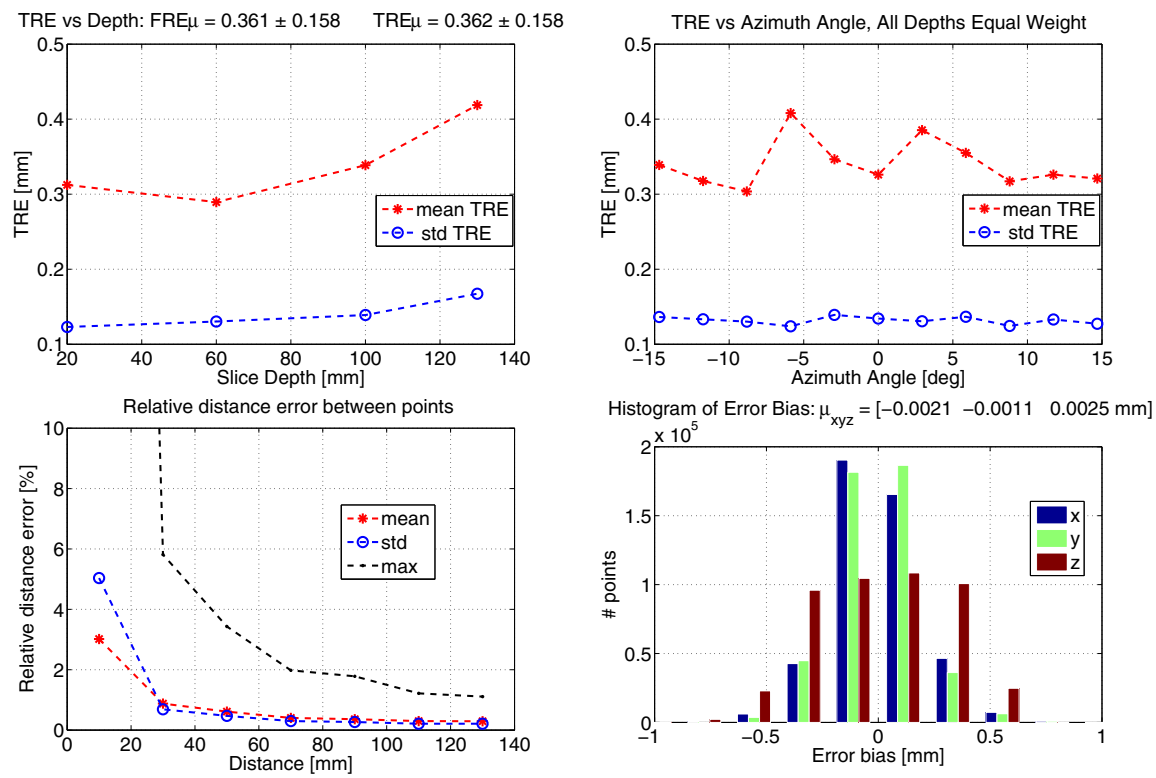

Fig. 4. Error measures from the first validation experiment: Top left: accuracy vs. depth. Top right: accuracy vs. azimuth angle. Bottom left: Percent distance error vs. distance between two points. Bottom right. Histograms of error bias values in X, Y, Z.

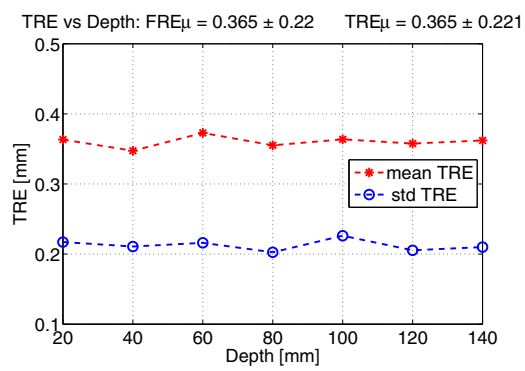

Fig. 5. Error measures from z-translation validation experiment: Accuracy vs. z-depth

stage translated the receiver in $1 \mathrm{~mm}$ increments along the center $\mathrm{z}$-axis from 10 to $140 \mathrm{~mm}$ for a total of 141 points per run. A total of 5110 data points were collected, representing 36 runs through the straight line. The system accuracy was evaluated with the same routine as the first experiment. The accuracy values are grouped by depth and shown in Fig. 5. The accuracy remains constant at $0.37 \mathrm{~mm}$ across all depths. Note that the average performance is same as before, but depth dependent variation is low due to constant sensor orientation. 

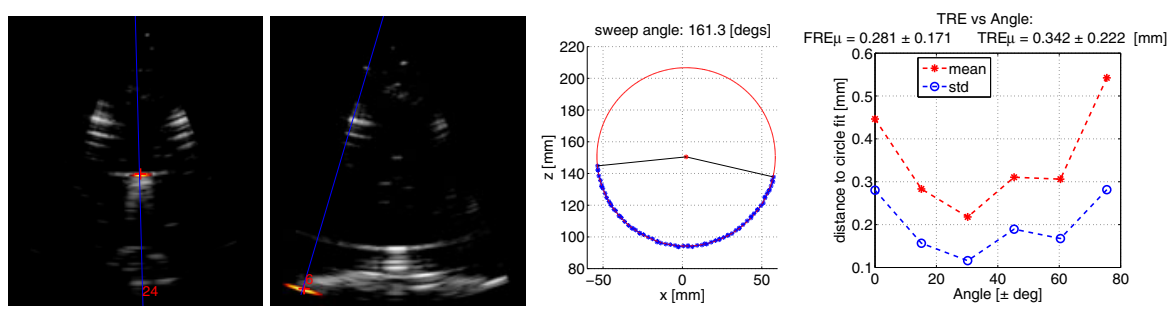

Fig. 6. Left: enhanced images of the hydrophone at approximately $0^{\circ}$ and $90^{\circ}$ incidence. Center: Recorded positions with circle fit. Right: TRE vs. hydrophone angle.

\subsection{Sensor Orientation}

A third experiment evaluated the system accuracy as a function of sensor angle. Here the receiver was mounted on a manual rotational stage with the center of rotation approximately $150 \mathrm{~mm}$ from the probe surface. The receiver tip was held at a radius of about $55 \mathrm{~mm}$ from the center of rotation and manually rotated from approximately $-80^{\circ}$ to $80^{\circ}$ for a total 73 data points. The collected data points were divided into 6 bins according to angle. Each bin of data was then selectively omitted so that 5 bins would be used for a circle-fit, while the omitted bin provided TRE values (distance to the circle). The accuracy is shown in Fig. 6. showing that the technique is robust to sensor orientations $(<0.6 \mathrm{~mm}$ error).

\subsection{Beating Heart Phantom}

The fourth and final experiment tested the ability to dynamically track a catheter prototype consisting of a $3 \mathrm{~mm}$ diameter by $5 \mathrm{~mm}$ long PZT cylinder sensor mounted on a $1.5 \mathrm{~m}$ thin-gauge coaxial cable [16]. It was tested with an inhouse beating heart phantom, a polymer replica with heart-like motion and US imaging appearance. Note in figure 1 the difficulty of tool detection without the image enhancement and tool tracking enabled. The received waveform was elongated at some angles likely due to the sensor's bandwidth and spatial sensitivity pattern. This had a deleterious effect on the overlay resolution (Fig. 1 right). Overall, this experiment qualitatively illustrates the added value of the tracking in a more realistic situation, with some amount of ultrasound aberration and absorption, and the presence of motion.

\section{Conclusion and Future Work}

We demonstrated real time US-based tool tracking on an FDA approved commercial scanner. Experiments indicate an accuracy of $0.36 \pm 0.16 \mathrm{~mm}$ and show robustness to varying imaging conditions. Because image enhancement occurs in US imaging space, the tool location and US image are always perfectly registered even in the face of image distortion. This method is non-disruptive where US is already part of the clinical workflow and requires no additional major capital equipment, thereby facilitating clinical adoption. However, this method requires that interventional tools incorporate US sensors and electrical connections safely 
and unobtrusively. This is an engineering task for future work. Future work also includes improving frame rate, pre-clinical and clinical validation and determining which US-guided procedures would benefit most from this technology.

\section{References}

1. Chin, K.J., Perlas, A., Chan, V.W., Brull, R.: Needle Visualization in UltrasoundGuided Regional Anesthesia: Challenges and Solutions. Reg. Anesth. Pain. Med. 33(6), 532-544 (2008)

2. O'Flynn, E.A., Wilson, A.R., Michell, M.J., et al.: Image-Guided Breast Biopsy: State-of-the-Art. Clin. Radiol. 65(4), 259-270 (2010)

3. Liveraghi, T.: Radiofrequency Ablation of Hepatocellular Carcinoma. Surg. Oncol. N. Am. 20(2), 281-299 (2011)

4. Balzer, R., Kelm, M., Kühl, H.P.: Real-Time Three-Dimensional Transoesophageal Echocardiography for Guidance of non-Coronary Interventions in the Catheter Laboratory. Eur. J. Echocardiogr. 10(3), 341-349 (2009)

5. Nichols, K., Wright, L.B., Spencer, T., Culp, W.C.: Changes in Ultrasonographic Echogenicity and Visibility of Needles with Changes in Angles of Insonation. J. Vasc. Interv. Radiol. 14(12), 1553-1557 (2003)

6. EchoTip®, Cook Medical, http: //www . cookmedical. com/wh/dataSheet. do?id=4480

7. Cheung, S., Rohling, R.: Enhancement of Needle Visibility in Ultrasound-Guided Percutaneous Procedures. Ultr. Med. Biol. 30(5), 617-624 (2004)

8. Sonosite Needle Visualization, http://www . sonosite.com/needlevisualization/

9. Idriss, S.F., Fronheiser, M.P.: Vibrating Interventional Device Detection Using Real-Time 3-D Color Doppler. IEEE Trans. Ultr. Ferr. Freq. Contr. 55(6), 1355$1362(2008)$

10. IGS Platforms, www . brainlab.com/art/2798/4/image-guided-surgery-platforms/

11. Breyer, B., Cikes, I.: Ultrasonically marked catheter-a method for positive echographic catheter position identification. Med. Biol. Eng. Comp. 22(3), 268-271 (1984)

12. Perrella, R.R., Kimme-Smith, C., Tessler, F.N., Ragavendra, N., Grant, E.G.: A new electronically enhanced biopsy system: value in improving needle-tip visibility during sonographically guided procedures. Am. J. Roentgenol. 158(1) (1992)

13. Vilkomerson, D., Lyons, D.: A system for ultrasonic beacon-guidance of catheters and other minimally-invasive medical devices. IEEE Trans. UFFC 44(2), 496-504 (1997)

14. Menz, V., Vilkomerson, D., Ren, J.F., Michele, J.J., Schwartzman, D.: Echocardiographic Transponder-Guided Catheter Ablation Feasibility and Accuracy. J. Interv. Card. Electrophysiol. 5(2), 203-209 (2001)

15. Merdes, C.L., Wolf, P.D.: Locating a catheter transducer in a three-dimensional ultrasound imaging field. IEEE Trans. Biomed. Eng. 48(12), 1444-1452 (2001)

16. Mung, J., Han, S., Yen, J.T., et al.: Design and in vitro evaluation of a realtime catheter localization system using time of flight measurements from seven 3.5 $\mathrm{MHz}$ single element ultrasound transducers towards abdominal aortic aneurysm procedures. Ultrasonics 51(6), 768-775 (2011)

17. Meyer, S.A., Wolf, P.D., et al.: Application of Sonomicrometry and Multidimensional Scaling to Cardiac Catheter Tracking. IEEE Trans. on Biomed. Eng. 44(11), 1061-1067 (1997) 